ARTICLE

\title{
The origin of efficient triplet state population in sulfur-substituted nucleobases
}

\author{
Sebastian Mai ${ }^{1}$, Marvin Pollum², Lara Martínez-Fernández ${ }^{3, \dagger}$, Nicholas Dunn², Philipp Marquetand ${ }^{1}$, Inés Corral ${ }^{3}$, \\ Carlos E. Crespo-Hernández ${ }^{2} \&$ Leticia González ${ }^{1}$
}

Elucidating the photophysical mechanisms in sulfur-substituted nucleobases (thiobases) is essential for designing prospective drugs for photo- and chemotherapeutic applications. Although it has long been established that the phototherapeutic activity of thiobases is intimately linked to efficient intersystem crossing into reactive triplet states, the molecular factors underlying this efficiency are poorly understood. Herein we combine femtosecond transient absorption experiments with quantum chemistry and nonadiabatic dynamics simulations to investigate 2-thiocytosine as a necessary step to unravel the electronic and structural elements that lead to ultrafast and near-unity triplet-state population in thiobases in general. We show that different parts of the potential energy surfaces are stabilized to different extents via thionation, quenching the intrinsic photostability of canonical DNA and RNA nucleobases. These findings satisfactorily explain why thiobases exhibit the fastest intersystem crossing lifetimes measured to date among bio-organic molecules and have near-unity triplet yields, whereas the triplet yields of canonical nucleobases are nearly zero.

\footnotetext{
${ }^{1}$ Institute of Theoretical Chemistry, Faculty of Chemistry, University of Vienna, Währinger Straße 17, Vienna 1090, Austria. ${ }^{2}$ Center for Chemical Dynamics and Department of Chemistry, Case Western Reserve University, 10900 Euclid Avenue, Cleveland, Ohio 44106, USA. ${ }^{3}$ Universidad Autónoma de Madrid, Departamento de Química, Cantoblanco, Madrid 28049, Spain. †Present Adress: Istituto Biostrutture e Bioimmagini-Consiglio Nazionale delle Ricerche, Via Mezzocannone 16, Napoli I-80134, Italy. Correspondence and requests for materials should be addressed to I.C. (email: ines.corral@uam.es) or to C.E.C.-H. (email: carlos.crespo@case.edu) or to L.G. (email: leticia.gonzalez@univie.ac.at).
} 
C hemically modified nucleobases provide DNA and, in particular, RNA with additional functions that are only beginning to be discovered ${ }^{1}$. Thiobases, where the oxygen atom of a carbonyl group is substituted by a sulfur atom, have been detected in transfer-RNA ${ }^{2,3}$, and the lack of some of these substituted nucleobases can lead to metabolic diseases in the mitochondria ${ }^{4}$. Thiobases are also employed in DNA-based nanotechnology ${ }^{5}$, in photocrosslinking studies ${ }^{6,7}$, in pharmacology 8,9 , and-due to their interesting photophysical properties-in photo-chemotherapy ${ }^{10-12}$. In fact, thiobases have recently been demonstrated as effective photo-chemotherapeutic agents for the treatment of skin cancer in tissue biopsies ${ }^{13}$ as well as of bladder cancer in animal models ${ }^{11}$, and are being developed for clinical applications ${ }^{14}$.

The canonical nucleobases absorb primarily in the ultraviolet- $\mathrm{C}$ range $(100-280 \mathrm{~nm})$. In contrast, substitution of the oxygen atom by sulfur induces a significant red-shift in the absorption spectrum, making thiobases effective absorbers of ultraviolet-B $(280-315 \mathrm{~nm})$ and ultraviolet-A $(315-400 \mathrm{~nm})$ radiation ${ }^{15}$. An intriguing observation is that, while canonical nucleobases efficiently return to the electronic ground state following electronic excitation ${ }^{16,17}$, thiobases populate long-lived, reactive triplet states in near-unity yields ${ }^{15}$ - a property that is exploited in their photonic applications. The photostability of canonical nucleobases is ascribed to ultrafast relaxation mechanisms that convert the photon energy into vibrational motion ${ }^{17}$. Although intense research activity has been aimed at determining time scales and relaxation pathways in thiobases by using time-resolved spectroscopic techniques ${ }^{12,18-24}$ and theoretical methods ${ }^{25-32}$, there exists no physical explanation of the electronic and structural factors that favour the formation of reactive triplet states instead of ultrafast relaxation to the ground state.

In this work, we provide a rationale for the origin of efficient population of triplet states in thiobases versus the ultrafast recovery of the ground state in canonical nucleobases. We use 2-thiocytosine $(2 \mathrm{tC})$ as a testbed to understand how thionation leads to a strong stabilization of different parts of the excited-state potential energy surfaces (PESs), which favours intersystem crossing (ISC) to the triplet manifold over internal conversion to the electronic ground state. Not only is $2 \mathrm{tC}$ specifically relevant for many medicinal applications-due to its anti-cancer ${ }^{33}$, anti-viral $^{34}$, anti-bacterial ${ }^{35}$, anti-microbial ${ }^{36}$ and cytotoxic activities $^{37}$ - but it is the only pyrimidine thiobase derivative that has not yet been investigated using time-resolved techniques ${ }^{15}$. By filling this gap we are able to deliver a comprehensive explanation of the remarkable photophysics of thiobases, providing design criteria for the synthesis of prospective drugs.

\section{Results}

Photochemical mechanism of 2-thiocytosine. A combination of steady-state and femtosecond transient absorption spectroscopy with ab initio computations and nonadiabatic dynamics is used to elucidate the excited-state dynamics of 2tC. Femtosecond transient absorption spectra (fs-TAS) were recorded exciting with UV-B $(308 \mathrm{~nm})$ and UV-A $(321 \mathrm{~nm})$ wavelengths, which correspond to the low-energy tail of the steady-state absorption spectrum (see Supplementary Fig. 1). According to calculations (see Supplementary Fig. 2, Supplementary Table 1 and Supplementary Note 1), the UV-A or UV-B wavelengths excite the lowest-energy ${ }^{1} \pi_{s} \pi^{*}$ state (the $S_{2}$ at the equilibrium geometry) or the tail of the second ${ }^{1} \pi_{s} \pi^{*}$ state $\left(S_{4}\right)$, respectively. The TAS obtained after $308 \mathrm{~nm}$ excitation (Fig. 1, the TAS exciting at $321 \mathrm{~nm}$ is shown in Supplementary Fig. 3) shows an initial featureless rise in the absorbance covering the full spectral probe window from $-360 \mathrm{fs}$ until a time delay of $-120 \mathrm{fs}$ (that is, within the cross correlation of the pump and probe beams), when

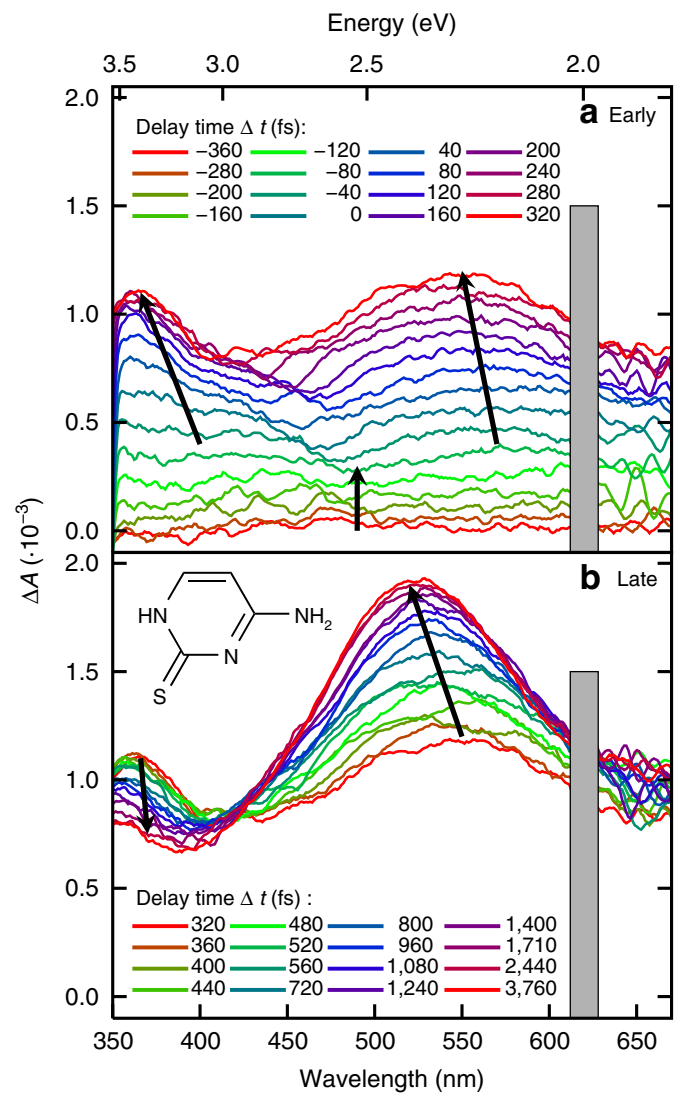

Figure 1 | Transient absorption spectra. (a) Early time delays ( -360 to $320 \mathrm{fs}$ ) and (b) later time delays (320 to 3,760 fs). The spectra were recorded in phosphate buffer solution $(\mathrm{pH} 7.4)$ using an excitation wavelength of $308 \mathrm{~nm}$. Arrows indicate the movement of the absorption bands with time. The grey boxes block the overtone band of the excitation light. The inset shows the molecular structure of 2-thiocytosine.

two distinct absorption maxima begin to emerge: one in the UV range $(350-400 \mathrm{~nm})$ and another in the visible range (around $580 \mathrm{~nm}$ ). These absorption bands continue to grow until $320 \mathrm{fs}$, at which point the UV band reaches its maximum. As the UV band decays, the visible band continues to increase in amplitude and blueshifts to $\lambda_{\max }=525 \mathrm{~nm}$ at $3.76 \mathrm{ps}$, with an apparent isosbestic point at $420 \mathrm{~nm}$. After $3.76 \mathrm{ps}$, no significant changes in the absorption maxima are observed for a time delay of up to $20 \mathrm{ps}$ (not shown), the maximum time delay investigated in this work.

The TAS data were subjected to a target and global fitting analysis. Two lifetimes $\left(\tau_{1}=210 \pm 50 \mathrm{fs}\right.$ and $\left.\tau_{2}=480 \pm 60 \mathrm{fs}\right)$ were needed to model the dynamics during the first $4 \mathrm{ps}$. Figure 2 shows the decay-associated spectra (DAS) and the fitted representative kinetic traces taken at the transient absorption maxima, that is, 355, 523 and $581 \mathrm{~nm}$ (see Supplementary Fig. 4 for the traces exciting at $321 \mathrm{~nm}$ ).

In order to interpret the TAS, and to deduce the relaxation pathways associated with the obtained lifetimes, the deactivation pathways out of the initially excited $S_{2}\left({ }^{1} \pi_{s} \pi^{\star}\right)$ state were calculated using the MS-CASPT2 method (multi-state complete active space second-order perturbation theory). TAS were simulated (Fig. 3a, the corresponding data is given in Supplementary Table 2) from the excited-state minima $\left({ }^{1} \pi_{s} \pi^{\star},{ }^{1} n_{s} \pi^{\star},{ }^{3} n_{s} \pi^{\star}\right.$ and $\left.{ }^{3} \pi_{s} \pi^{\star}\right)$ using a sum of Gaussians centred at the calculated transition energies, with the amplitudes proportional to the oscillator strengths. The ${ }^{1} \pi_{s} \pi^{*}$ state absorbs strongly around $363 \mathrm{~nm}$ and the ${ }^{3} \pi_{s} \pi^{\star}$ state dominates the visible part of the spectrum with two transitions around 441 and $546 \mathrm{~nm}$, 


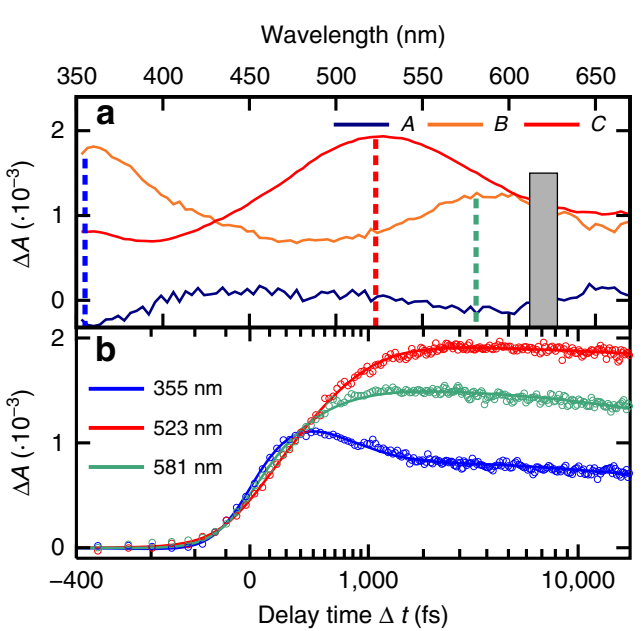

Figure 2 | Target and global analysis of the TAS. (a) Decay associated spectra obtained by globally fitting the transient absorption data to a twocomponent sequential kinetic model $(A$ and $B$ ) with a constant offset $(C)$. (b) Kinetic traces and fitted functions from the global fit analysis, taken at representative probe wavelengths, as indicated with coloured dashed lines in (a).

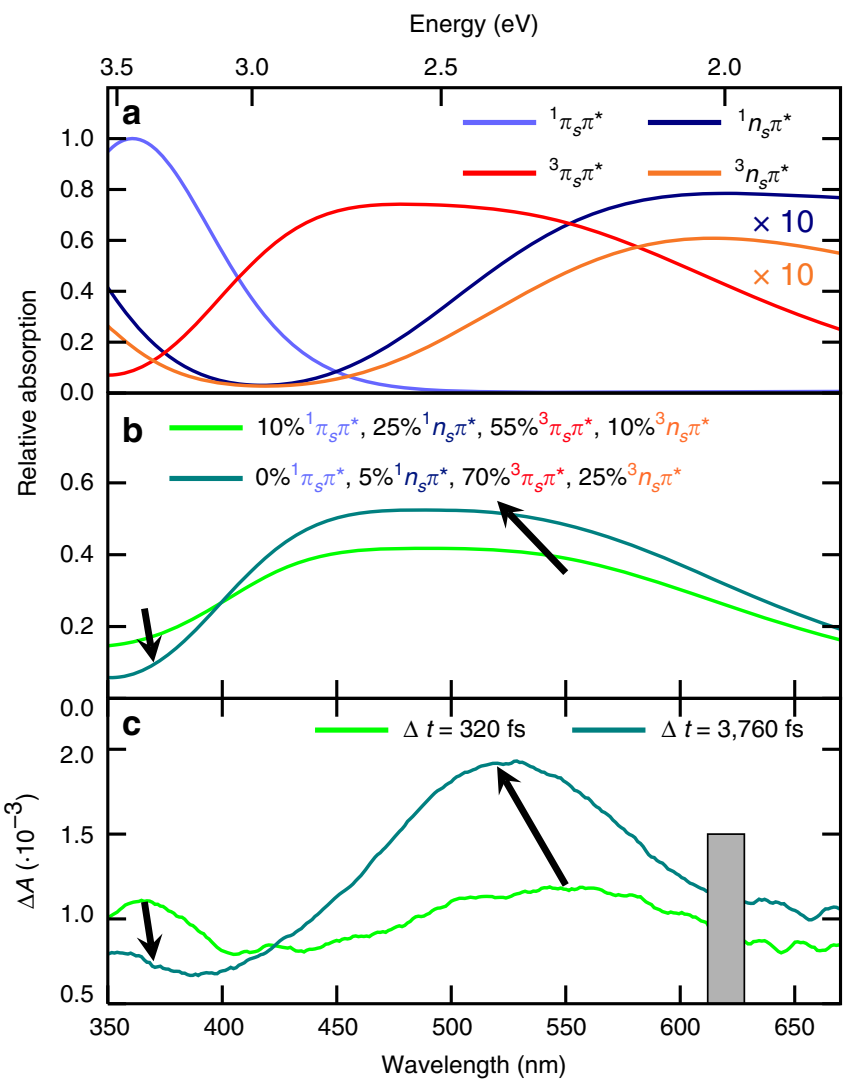

Figure 3 | Simulated absorption spectra of the excited states.

(a) Calculated absorption spectra from the given excited states at their respective minimum geometries (MS-CASPT2, convoluted using Gaussians with $0.7 \mathrm{eV}$ full-width at half-maximum). (b) Linear combinations of the calculated spectra with the given proportions. (c) The experimental transient absorption spectra at the indicated time delays.

giving rise to a broad absorption band. In contrast, the two $n_{s} \pi^{\star}$ states show a 10 -fold weaker absorbance compared with the $\pi_{s} \pi^{*}$ states. The absorption spectra of the singlet and triplet $n_{s} \pi^{\star}$ states are very similar, with transitions at 333 and $576 \mathrm{~nm}$ versus 333 and $574 \mathrm{~nm}$, respectively.

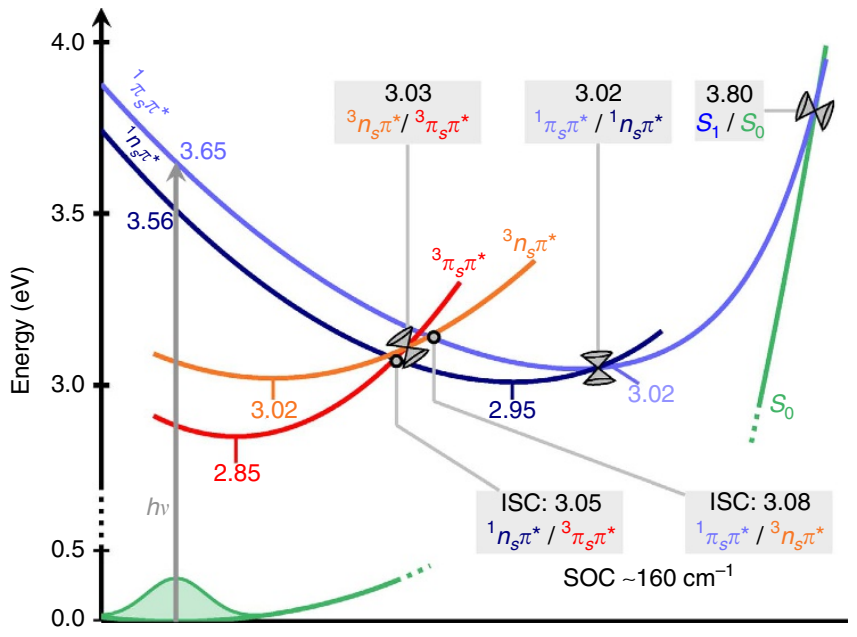

Figure 4 | Schematic representation of possible relaxation pathways. All energies were computed at the MS-CASPT2 level of theory and are given in eV. Additional information can be found in Supplementary Note 2.

Figure $3 \mathrm{~b}$ shows two linear combinations of the four calculated transient spectra harmonizing with the experimental transient spectra at selected time delays, that is, at $320 \mathrm{fs}$ (when the UV band reaches its maximum intensity) and 3,760 fs (when the visible band reaches its maximum). The per cent contribution of each excited-state absorption can readily explain both the decay in the UV region $(<400 \mathrm{~nm})$ and the increase of the visible band around $525 \mathrm{~nm}$. For comparison, the two corresponding experimental transient spectra are shown in Fig. 3c.

According to the calculations, the initial broad and featureless increase in absorption amplitude across the full spectral probe window until $-120 \mathrm{fs}$ (see also DAS $A$ in Fig. 2a) can be assigned to a linear combination of the singlet states with $\pi_{s} \pi^{\star}$ and $n_{s} \pi^{\star}$ characters. This assignment is further supported by dynamics simulations, which predict that a ${ }^{1} \pi_{s} \pi^{*} /{ }^{1} n_{s} \pi^{\star}$ conical intersection (CoIn) can be reached within $160 \mathrm{fs}$ upon excitation, from which the molecule populates simultaneously two singlet minima with ${ }^{1} n_{s} \pi^{\star}$ and ${ }^{1} \pi_{s} \pi^{\star}$ characters.

At a time delay of about $-80 \mathrm{fs}$, two distinct absorption bands begin to evolve, with maxima around 360 and $550 \mathrm{~nm}$, respectively (Fig. 1 and DAS $B$ in Fig. 2a). These absorption bands are assigned to a linear combination of the absorption from the ${ }^{1} \pi_{s} \pi^{*},{ }^{1} n_{s} \pi^{*},{ }^{3} n_{s} \pi^{*}$ and ${ }^{3} \pi_{s} \pi^{*}$ states (Fig. 3b). The UV absorption band matches most closely the spectrum computed for the ${ }^{1} \pi_{s} \pi^{\star}$ state, thus the subsequent decay in this region is likely associated with a decay of ${ }^{1} \pi_{s} \pi^{\star}$ population.

Finally, the TAS at a time delay of ca. 4 ps, which has an absorption band peaking at $525 \mathrm{~nm}$ (Fig. 1 and DAS $C$ in Fig. 2a), compares satisfactorily with the spectrum calculated for the ${ }^{3} \pi_{s} \pi^{*}$ state, possibly with some contribution from the ${ }^{3} n_{s} \pi^{*}$ state (Fig. 3b).

On the basis of static MS-CASPT2 calculations, the relaxation pathways sketched in Fig. 4 can be postulated (see Supplementary Table 3 for the corresponding data). After one-photon absorption (308 or $321 \mathrm{~nm}$ ) the ${ }^{1} \pi_{s} \pi^{*}$ state at $3.65 \mathrm{eV}$ is populated $\left(S_{2}\right.$ at the Franck-Condon geometry). A barrierless pathway leads to the ${ }^{1} \pi_{s} \pi^{\star} /{ }^{1} n_{s} \pi^{\star}$ CoIn at $3.02 \mathrm{eV}$, which connects to two different singlet state minima with ${ }^{1} \pi_{s} \pi^{*}(3.02 \mathrm{eV})$ or ${ }^{1} n_{s} \pi^{\star}(2.95 \mathrm{eV})$ character. From these two minima, several relaxation pathways are plausible. Relaxation to the ground state involves overcoming energy barriers of at least $0.8 \mathrm{eV}$, making this process unlikely. Instead, two singlet-triplet $\left({ }^{1} n_{s} \pi^{*} / 3 \pi_{s} \pi^{*}\right.$ and $\left.{ }^{1} \pi_{s} \pi^{*} / 3 n_{s} \pi^{*}\right)$ minimum-energy crossing points (MECPs) located at 3.05 and at $3.08 \mathrm{eV}$, respectively, are accessible due to their small energy 
barriers and associated spin-orbit couplings of up to $170 \mathrm{~cm}^{-1}$. Energetically and geometrically close to these MECPs lies a CoIn between the ${ }^{3} n_{s} \pi^{*}$ and ${ }^{3} \pi_{s} \pi^{*}$ states, at an energy of $3.03 \mathrm{eV}$. Hence, there exists a three-state near-degeneracy region involving the lowest singlet and the two lowest triplet states. From the ${ }^{3} n_{s} \pi^{*} / 3 \pi_{s} \pi^{*}$ CoIn, the system can relax either to the ${ }^{3} n_{s} \pi^{*}$ minimum at $3.02 \mathrm{eV}$ or to the ${ }^{3} \pi_{s} \pi^{*}$ minimum at $2.85 \mathrm{eV}$.

A time-resolved interpretation of the relaxation mechanism of $2 \mathrm{tC}$ is obtained by performing surface-hopping nonadiabatic simulations using the SHARC method (surface hopping including arbitrary couplings $)^{38}$. Associated energies, gradients and relevant couplings are obtained at the multi-reference configuration interaction including single excitations (MRCIS) level of theory (see Supplementary Fig. 5 and Supplementary Note 2 for validation of the method). Figure 5 shows the excited-state populations based on 137 trajectories and the result of monoexponential fitting. The relaxation pathways in the dynamics simulations involve mainly four electronic stateslabelled according to their energetic ordering at each geometry as $S_{1}, S_{2}, T_{1}$ and $T_{2}$-as foreseen by the quantum chemical calculations. These states encompass the ${ }^{1} n_{s} \pi^{\star},{ }^{1} \pi_{s} \pi^{*},{ }^{3} n_{s} \pi^{*}$ and ${ }^{3} \pi_{s} \pi^{\star}$ characters, but note that due to state crossing and mixing, no one-to-one correspondence between the $S_{i} / T_{i}$ labels and the characters exists beyond the Franck-Condon geometry.

After excitation, the population in the $S_{2}$ state decays to the $S_{1}$ state with a lifetime of $160 \mathrm{fs}$ (Supplementary Table 4) - a value that might be slightly overestimated due to a small barrier to reach the $S_{2} / S_{1}$ crossing $(0.13 \mathrm{eV})$, which is present with MRCIS but not with the more accurate MS-CASPT2 method (Fig. 4). The population of the $S_{1}$ state rises to a maximum of about $30 \%$ during the first $200 \mathrm{fs}$ due to population transfer from the $S_{2}$ state. Afterwards, 2tC undergoes ISC to the triplet manifold with an average time constant of $\sim 250 \mathrm{fs}$. The population of the $S_{1}$ state amounts to only $13 \%$ after 1 ps.

The most important ISC pathways predicted by the dynamics simulations are $S_{1} \rightarrow T_{2}$ and $S_{1} \rightarrow T_{1}$, with a minor contribution from $S_{2} \rightarrow T_{2}$. The characters of the triplet states $T_{1}$ and $T_{2}$ are strongly mixed and cannot be assigned as strictly ${ }^{3} \pi_{s} \pi^{*}$ or ${ }^{3} n_{s} \pi^{*}$ during the dynamics simulations. It is precisely this mixed wavefunction character that leads to large spin-orbit couplings between $S_{1}$ and both triplet states, explaining why both $S_{1} \rightarrow T_{1}$ and $S_{1} \rightarrow T_{2}$ transitions are possible, and why ISC is very efficient. According to the simulations, $T_{2}$ is populated faster (in $\sim 100 \mathrm{fs}$ ) than $T_{1}$ (in $330 \mathrm{fs}$ ) because the average $S_{1} \rightarrow T_{2}$ energy gap is smaller than the $S_{1} \rightarrow T_{1}$ gap. Note that the spin-orbit couplings vary in these regions but are on average $\sim 160 \mathrm{~cm}^{-1}$ between $S_{1}$ and $T_{2}$ as well as $50 \mathrm{~cm}^{-1}$ between $S_{1}$ and $T_{1}$. However, the $T_{1}$ state receives most of the population within 1 ps due to subsequent $T_{2} \rightarrow T_{1}$ internal conversion, leading to an average ISC lifetime of $250 \mathrm{fs}$. This value is in full agreement with the lifetime

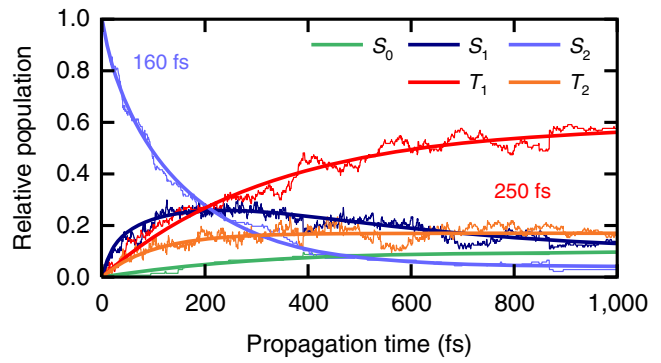

Figure 5 | Excited-state populations from surface hopping simulations. Simulations were performed with the SHARC method coupled to the MRCIS electronic structure method. Thin lines represent the simulated electronic populations, thick lines show the fitted functions. of $210 \pm 50$ fs obtained experimentally. After 1 ps, $74 \%$ of the total population resides in either of the triplet states, and the remaining $16 \%$ of the population in the excited singlet manifold likely undergoes ISC at later times. A small fraction of the trajectories (10\%) returned to the ground state because MRCIS underestimates the energy of the relevant $S_{1} / S_{0}$ CoIns, as compared with MS-CASPT2 (Supplementary Table 3 ). This ground-state repopulation should be regarded as an artefact of employing MRCIS in the dynamics simulations and we therefore estimate a $>90 \%$ total ISC yield from the simulations.

From a structural point of view, there are no large out-of-plane deformations during the dynamics as anticipated from the similarity and quasi-planar nature of all excited-state minima (Supplementary Note 3). During the $S_{2} \rightarrow S_{1}$ internal conversion, there is a bond inversion within the conjugated double bond system of the pyrimidine ring. As the trajectories relax to $S_{1}$ and the triplet states, ring puckering increases, probably due to the increased available energy or due to a more flexible pyrimidine ring in these states. The triplet minima are structurally similar to the singlet minima and thus motion on the $T_{1}$ and $T_{2}$ PESs cannot be distinguished from motion on the $S_{1}$ PES. In fact, the structural similarity is one of the factors leading to the efficient ISC observed in this work.

In summary, the experimental and theoretical results suggest that excitation of $2 \mathrm{tC}$ to the lowest-energy ${ }^{1} \pi_{s} \pi^{*}$ state $\left(S_{2}\right)$ leads to very efficient ISC to the ${ }^{3} \pi_{s} \pi^{*}\left(T_{1}\right)$ state in three general steps, which are schematically depicted in Fig. 6. The first step is the relaxation from the vertically excited $S_{2}\left({ }^{1} \pi_{s} \pi^{\star}\right)$ state to the $S_{1}$ state through an $S_{2} / S_{1}$ CoIn. After transition from the $S_{2}$ to the $S_{1}$ state, the system populates the $S_{1}$ PES, which in some regions of the configurational space has dominant ${ }^{1} \pi_{s} \pi^{*}$ character and in other regions ${ }^{1} n_{s} \pi^{*}$ character, according to MS-CASPT2 calculations. This is also consistent with the observation of a band assigned to the ${ }^{1} \pi_{s} \pi^{*}$ state in the TAS at short time delays. The second step is ultrafast ISC from the $S_{1}$ state to the triplet manifold ( $T_{2}$ and $T_{1}$ states). In the time-resolved experiments, this process leads to the appearance of an absorption band assigned to the ${ }^{3} \pi_{s} \pi^{*}$ state. This band begins to appear at approximately the same time as the ${ }^{1} \pi_{s} \pi^{*}$ absorption band, indicating that internal conversion in the singlet manifold and ISC occur on very similar time scales. The experimental time constant $\tau_{1}$ for this second step is $210 \pm 50 \mathrm{fs}$, in close agreement to the ISC lifetime of $250 \mathrm{fs}$ observed theoretically. As the calculations show, the three-state near-degeneracy between $S_{1}, T_{2}$ and $T_{1}$, together with the large spin-orbit matrix elements, are responsible for the ultrafast ISC lifetime of $2 \mathrm{tC}$. Finally, the third step is internal conversion from the $T_{2}$ to the $T_{1}$ state and subsequent vibrational energy redistribution to populate the lowest-energy minimum of the $T_{1}\left({ }^{3} \pi_{s} \pi^{*}\right)$ state. The second experimental time constant of $480 \pm 60 \mathrm{fs}$ is attributed to the latter step, together with residual ISC, as suggested by the decay of the UV transient absorption band. The eventual relaxation from the $T_{1}$ state to the ground state appears to involve significant energetic barriers and does not play a role on the time scales

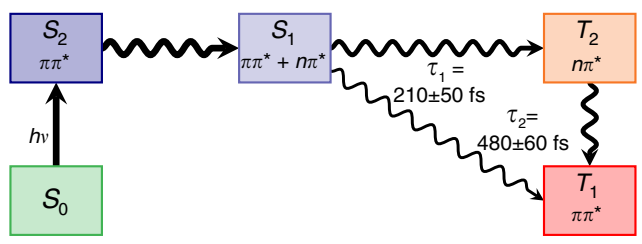

Figure 6 | General relaxation mechanism of 2-thiocytosine. The scheme is based on the presented experimental and computational data. The $\tau_{1}$ and $\tau_{2}$ are the experimental time constants. 
considered in this work, in agreement with the long-lived nature of the $T_{1}$ state of thiobases in solution ${ }^{15}$.

Thiobases versus canonical bases. Having revealed the electronic relaxation mechanisms of $2 \mathrm{tC}$, we are now in a favourable position to compare the structural and electronic features of the PESs in the thiobases and the canonical nucleobases, in order to address the intriguing question as to why they are so photophysically different. To this end, Table 1 collects the energetic parameters for cytosine $(\mathrm{C})$, uracil $(\mathrm{U})$ and guanine $(\mathrm{G})$, and those of the corresponding thiobase analogues that have been investigated thus far-since the relative energies of the states minima and the $S_{1} / S_{0}$ surface crossings are strongly correlated to whether or not a nucleobase exhibits ultrafast relaxation to the ground state ${ }^{39}$.

The first conspicuous fact is that sulfur-substitution significantly lowers the energies of the singlet state minima. Both ${ }^{1} n \pi^{*}$ and ${ }^{1} \pi \pi^{*}$ state minima are stabilized by up to $1 \mathrm{eV}$ for all three thiobases $(2 \mathrm{t} C, 2 \mathrm{tU}, 6 \mathrm{tG})$ in comparison with the corresponding canonical nucleobase. This stabilization is well-known in thiocarbonyl compounds ${ }^{40}$ and thiobases ${ }^{15,30}$. However, it is surprising that the energy of the most accessible (that is, lowest-energy) CoIn of each molecule stays approximately constant upon thionation $(3.8 / 3.8 \mathrm{eV}$ for $\mathrm{C} / 2 \mathrm{tC}$, $4.0 / 4.0 \mathrm{eV}$ for $\mathrm{U} / 2 \mathrm{tU}$ and $4.3 / 3.8 \mathrm{eV}$ for $\mathrm{G} / 6 \mathrm{tG}$ ). As a consequence, canonical nucleobases exhibit efficient groundstate recovery after excitation, whereas the population of the thiobases rapidly decays to deep singlet state minima. The presence of one or more triplet states with energies similar to the singlet state minima and with large spin-orbit couplings facilitates subsequent ISC in the thiobases. While the energetic stabilizations can be correlated directly with the excited-state behaviour of the thiobases, it is not straightforward to rationalize why thionation lowers the energies of the states' minima so strongly, but has little impact on the energies of relevant CoIns.

We argue that a plausible explanation relates to the moiety of the molecule where the electronic excitation is localized. We focus on whether the excitation (that is, the excited electron or, more importantly, the hole left behind) is localized significantly on the (thio)carbonyl group or on the aromatic ring. States characterized by an excitation localized on the chalcogene atom are expected to stabilize considerably upon thionation because it takes less energy to excite an electron from an $n$ or a $\pi$ orbital of a sulfur atom into the $\pi^{*}$ system than from an $n$ or $\pi$ orbital of an oxygen atom (compare the lower ionization potential of $\mathrm{S}$ versus $\mathrm{O})^{41}$. Conversely, if the electronic excitation is localized primarily on the pyrimidine ring (that is, electronic excitation from pyrimidine $\pi$ to pyrimidine $\pi^{*}$ ) no stabilization is expected upon thionation. Using the TheoDORE program ${ }^{42}$, the electronic wave functions were analysed to assign the localization of the relevant $S_{0} \rightarrow S_{1}$ excitations. The results are shown in Table 1 (the corresponding difference densities of the excitations can be found in Supplementary Fig. 6, the numeric data in Supplementary Table 5). Remarkably, the stabilization of the excited-state minima of the thiobases can now be easily explained. Since the $n \pi^{\star}$ states are always localized on the carbonyl/thiocarbonyl group, the $n \pi^{*}$ state minima are stabilized strongly upon thionation by $\sim 1 \mathrm{eV}$. Similar arguments explain why the $\pi \pi^{*}$ states stabilize, but to lesser extent because they are at least partially delocalized over the aromatic rings.

In order to rationalize the energy shifts of the CoIns upon thionation, we consider $\mathrm{C}$ and $2 \mathrm{tC}$ as the initial example. Several different $S_{1} / S_{0}$ CoIns have been reported for C (Table 1) $)^{43-49}$ These CoIns have different electronic character and hence we expect different localization of the excitations. The excitation within the semiplanar CoIn is localized on the chalcogen atom and thus stabilized in $2 \mathrm{tC}$. However, the ethylenic CoIn (which is the lowest-energy CoIn of $\mathrm{C}$, and thus primarily responsible for its ultrafast relaxation) is not stabilized because its excitation is centred on the pyrimidine ring. The amino-out-of-plane (oop- $\mathrm{NH}_{2}$ ) CoIn is stabilized slightly (by $0.3 \mathrm{eV}$ ). Although the semiplanar CoIn is also stabilized by $1.4 \mathrm{eV}$ in $2 \mathrm{tC}$ and becomes the lowest-energy CoIn, it is still too high in energy compared with the ${ }^{1} n \pi^{*}$ minimum, precluding ultrafast internal conversion

Table 1 | Relative energies of stationary points and crossings for $2 \mathrm{tC}, 2 \mathrm{tU}, 6 \mathrm{tG}$, and their parent nucleobases.

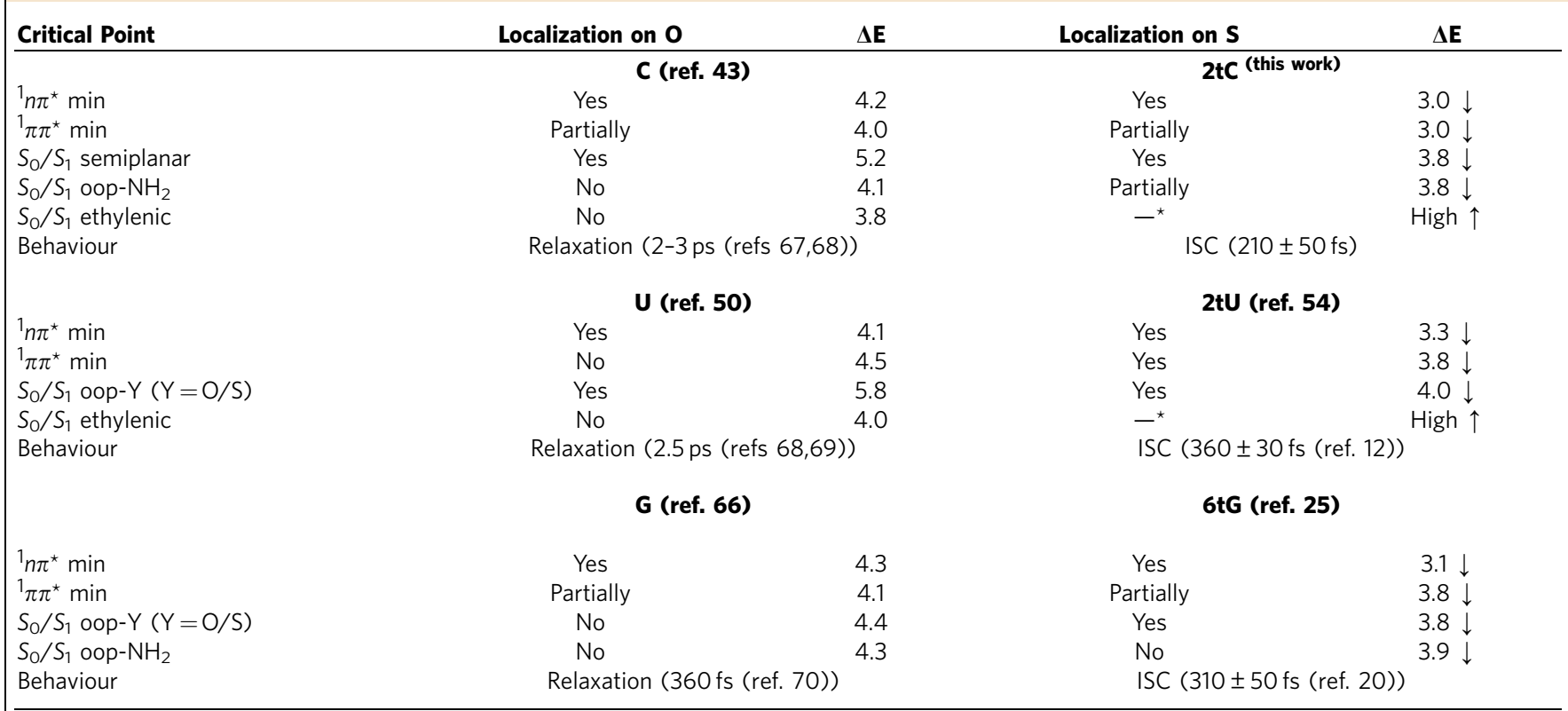

All energies $(\Delta E)$ are given in $\mathrm{eV}$ above the ground state minimum of the respective molecule. The classification whether the $S_{0} \rightarrow S_{1}$ excitation is localized on the chalcogen atom $(Y=O / S)$ is based on the density plots presented in Supplementary Fig. 6 and Supplementary Table 5. These plots were calculated at the CASSCF $(14,10)$ level of theory $($ CASSCF $(18,13)$ for G and 6tG). ${ }^{\star}$ Not reported in the literature, but presumed to have no localization on $\mathrm{S}$ in analogy to the equivalent Coln in the parent nucleobase. 
to the ground state and favouring instead rapid decay to the ${ }^{1} n \pi^{\star}$ minimum and subsequent ISC to the triplet manifold.

Satisfactorily, a similar rationale applies to the $\mathrm{U} / 2 \mathrm{tU}$ pair. According to calculations, $U$ relaxes primarily through the ethylenic $\mathrm{CoIn}^{50-53}$, with a twist at the carbon-carbon double bond, whereas the oxygen-out-of-plane (oop-O) CoIn ${ }^{50,52}$ is much higher in energy. In $2 \mathrm{tU}$, on the other hand, the oop-S CoIn is stabilized, whereas the ethylenic CoIn is much higher in energy $^{26,54}$. The stabilization of the oop-S CoIn is, however, not sufficient enough to provide a fast relaxation channel to the ground state ${ }^{54}$. To our knowledge, there are no calculations on 2-thiothymine, but we expect that similar arguments hold for the thymine/2-thiothymine pair, because the methyl group is not expected to affect the electronic structure significantly.

The CoIns in the G/6tG pair also follow the discussed trend: the oop-S CoIn is stabilized more than the oop- $\mathrm{NH}_{2}$ CoIn-although the effect is less pronounced than in the pyrimidine bases. Ground-state relaxation from the ${ }^{1} \pi \pi^{*}$ minimum in $6 \mathrm{tG}$ would be energetically possible, but the shape of the PES favours the population of the ${ }^{1} n \pi^{\star}$ minimum instead, from where all possible deactivation pathways to $S_{0}$ involve very large energy barriers.

From the above analysis, it can be proposed that thionation of the nucleobases stabilizes all states in which the electronic excitation is primarily localized on the sulfur atom (for example, $\left.n_{s} \pi^{*}, \pi_{s} \pi^{*}\right)$, whereas the other states $\left(n_{N} \pi^{*}, \pi_{\text {ring }} \pi^{*}\right)$, and their corresponding CoIns, are not stabilized to the same extent. This leads to the conclusion that the intrinsic relaxation pathways in the canonical nucleobases-which involve extensive ring puckering but no extensive motion of the carbonyl group-are blocked in the thiobases because they are too high in energy relative to the considerably stabilized excited-state minima. Although there exist $S_{0} / S_{1}$ CoIns involving sulfur-localization that are also stabilized $25,29,54$, they are too high in energy with respect to the excited-state minima and do not play a role in the deactivation.

Finally, localization of the excitation on the sulfur atomic orbitals also enhances spin-orbit coupling. This is because the spin-orbit operator is of short range nature ${ }^{55}$ and depends on the atomic number, thus making this coupling largest if the excitations are localized on the heaviest atom. Further enhancement of population transfer from the singlet to the triplet manifold is due to the smaller singlet-triplet energy gaps in the thiobases compared with the canonical nucleobases because heavy atom substitution often leads to an increased density of states.

\section{Discussion}

Using transient absorption spectroscopy and theoretical calculations, we demonstrated that photoexcited 2-thiocytosine populates the triplet manifold on a femtosecond timescale and with close-to-unity triplet yield. Its relaxation after excitation proceeds in three general steps, which partially overlap: (i) barrierless deactivation from the $S_{2}$ to the $S_{1}$ state; (ii) ISC to the triplet manifold with a time constant of $210 \pm 50 \mathrm{fs}$, enhanced by the presence of a three-state near-degeneracy region involving the $S_{1}, T_{2}$ and $T_{1}$ states; and (iii) internal conversion to the $T_{1}$ state. The latter step, together with some residual ISC is responsible for a time constant of $480 \pm 60 \mathrm{fs}$. More importantly, we have rationalized and generalized the influence of thionation on the excited-state dynamics of the canonical nucleobases. While it is generally recognized that thionation leads to a strong decrease in excited-state energies, here we show that the extent of this stabilization differs significantly in different parts of the PESs. Furthermore, we have shown that the stabilization depends sensitively on the electronic wavefunction character and favours wave functions where the excitation is localized on the sulfur atom. Hence, excited-state minima (for example, ${ }^{1} n_{s} \pi^{*}$ ) and conical intersections involving the thiocarbonyl group are stabilized upon thionation, whereas the conical intersections intrinsic to the pyrimidine skeleton are not. This observation explains satisfactorily the remarkably different behaviour of thiobases compared with their parent nucleobases and provides design criteria for the synthesis of prospective photochemotherapeutic drugs.

\section{Methods}

Materials. 2-Thiocytosine (97\%, Sigma-Aldrich), sodium dihydrogenphosphate (99.0\%, Sigma-Aldrich), and sodium hydrogenphosphate ( $99.0 \%$, Sigma-Aldrich) were used as received. PBS solutions were prepared using $0.15 \mathrm{~g}$ of sodium dihydrogenphosphate and $0.27 \mathrm{~g}$ of sodium hydrogenphosphate dissolved in $200 \mathrm{ml}$ of ultrapure water to give $\mathrm{pH} 7.4$ aqueous phosphate buffer with a total phosphate concentration of $16 \mathrm{mM}$.

Femtosecond transient absorption spectroscopy. Femtosecond broadband transient absorption spectroscopy was performed using a Libra-HE pulsed laser system (Coherent, Inc.), which produces $800 \mathrm{~nm}, 100 \mathrm{fs}$ pulses with a total output power of $4 \mathrm{~W}$. A small fraction of this fundamental beam $(<2 \mathrm{~mW})$ was split off and focused into a continuously moving $2 \mathrm{~mm} \mathrm{CaF}_{2}$ crystal to generate the white light continuum probe pulses $(320-710 \mathrm{~nm})$. The remainder of the fundamental beam was directed through an optical parametric amplifier (TOPAS-800, Light Conversion, Ltd.) to generate the excitation (that is, pump) pulses at either 308 or $321 \mathrm{~nm}$. All other wavelengths were removed from the excitation beam using a reflective wavelength filter and a Glan-Taylor polarizer.

The spectrometer (Helios, Ultrafast Systems, LLC.) uses an optical delay line to delay the probe pulses in time with respect to the pump. The pump power was attenuated to $1 \mu \mathrm{J}$ at the sample and its polarization was randomized using a depolarizing plate in order to prevent contribution of rotational effects to the dynamics. At each delay time the spectrum of the probe is collected with and without pumping the sample in order to produce the difference spectra $(\Delta \mathrm{A})$.

$2 \mathrm{tC}$ solutions were prepared at concentrations of $5-8 \mathrm{mM}$ in $\mathrm{pH} 7.4$ aqueous PBS. All transient absorption experiments were performed in $2 \mathrm{~mm}$ optical path length quartz cuvettes (Starna Cells, Inc.). Sample solutions were stirred

continuously throughout data collection and replaced with fresh sample, from the same stock, every 5-7 scans so that all scans had no more than $5 \%$ degradation as determined by the steady-state absorption. A home-made LabView program (National Instruments, Inc.) was used to correct all transient absorption data for group velocity dispersion of the white light probe ${ }^{56}$. Global and target analysis of the data were performed using a sequential kinetic model ${ }^{22}$, convoluted with the instrument response function fixed at $200 \mathrm{fs}$, as determined by the coherence signal of neat methanol ${ }^{12}$

The sequential kinetic model included two lifetimes:

$$
A \stackrel{\tau_{1}}{\longrightarrow} B \stackrel{\tau_{2}}{\longrightarrow} C .
$$

The spectrum of the third component $C$ can be considered as an offset and represents the transient species that has not decayed during the first $20 \mathrm{ps}$ investigated in this work.

The results of five separate experiments, three exciting at $308 \mathrm{~nm}$ and two exciting at $321 \mathrm{~nm}$, were analysed. The target and global fitting analysis was performed independently for each of the five experiments in order to estimate the uncertainty of the lifetimes. Up to 91 kinetic traces from the broadband transient absorption data were used in this analysis. The uncertainties are reported as twice the standard deviation. The DAS are extracted from the global fit of the data and correspond to the wavelength-dependent amplitudes for each of the lifetime components in the kinetic traces.

Stationary quantum chemistry. According to Kasha's rule, the relaxation from $S_{4}$ via $S_{3}$ to $S_{2}$ should be ultrafast. This is in agreement with the experimental fs-TAS results, which showed that excitation at $321 \mathrm{~nm}$ (mainly to $S_{2}$ ) and excitation at $308 \mathrm{~nm}$ (which also excites $S_{4}$ ) induce the same excited-state dynamics. Hence, the computational investigations focused on the relaxation dynamics starting from $S_{2}$.

Minima of the PESs were first optimized at the CASSCF $(14,10) / A N O-S-V D Z P$ level of theory, whereas for the optimization of CoIns and MECPs CASSCF $(14,10)$ / 6-31G* was used. Using CASSCF $(14,10) / A N O-S-V D Z P$, all minima and crossing points were connected with minimum-energy paths to check for the absence of energetic barriers. If a minimum-energy path successfully finishes from a surface crossing to a minimum, it implies that there are no energetic barriers in between. The minima, CoIns and MECPs were then reoptimized at the MS-CASPT2/ANOL-VTZP level of theory using the methodologies described in refs. 57, 58 and the ORCA optimizer ${ }^{59}$. For all MS-CASPT2 calculations, an imaginary level shift of 0.3 a.u. and the default IPEA shift of 0.25 a.u. has been used. All calculations were conducted in the gas phase and using MOLCAS 7.8 (ref. 60), except for the 
CASSCF optimizations of the CoIns and MECPs, where MOLPRO 2009 (ref. 61) was used.

The levels of theory used in the various calculations, including the numbers of averaged states, are also compiled in Supplementary Table 6. The active space or reference space orbital composition is presented in Supplementary Fig. 7. All calculations were performed with the amino-thion tautomer of 2-thiocytosine, which is justified in Supplementary Fig. 8 and Supplementary Table 7.

All optimized geometries are reported in Supplementary Note 3.

While MS-CASPT2 is, in many cases, an excellent method to describe excited states, it cannot be yet routinely employed in dynamics simulations, due to the lack of efficient implementations of analytical MS-CASPT2 nuclear gradients. Hence, all stationary quantum chemistry calculations were repeated with MRCIS (as specified in 'nonadiabatic dynamics simulations') to scrutinize the accuracy of the MRCIS PESs employed in the dynamics simulations versus the MS-CASPT2 ones. See Supplementary Fig. 5, Supplementary Table 3 and Supplementary Note 2 for further discussions.

Nonadiabatic dynamics simulations. The on-the-fly electronic structure level of theory for the nonadiabatic dynamics simulations was MRCIS with a reference space of CAS $(6,5)$ type. The orbitals were taken from previous $\operatorname{CASSCF}(10,8) /$ cc-pVDZ calculations (see Supplementary Fig. 7 for the orbitals used), with state-averaging over four singlet and two triplet states (note that the number of states in the MRCIS calculations does not need to equal the number of states in the SA-CASSCF calculations). The CAS $(6,5)$ has been created removing the least occupied $\pi^{*}$-orbital and the most occupied two $\pi$-orbitals from the CAS $(10,8)$, and therefore the reduced reference space still contains all orbitals involved in the primary excitations of all the relevant states. Inner shells were kept frozen in the MRCIS stage of the calculations. All MRCIS calculations were conducted with COLUMBUS 7 (ref. 62), with integrals from MOLCAS ${ }^{60}$, which also provided scalar-relativistic one-electron integrals according to the Douglas-Kroll-Hess method and the spin-orbit integrals using atomic mean-field integrals ${ }^{55}$.

The surface hopping code SHARC was employed ${ }^{38}$. Surface hopping is an extension of classical molecular dynamics to include nonadiabatic transitions between excited states, and SHARC in turn is an extension of surface hopping to include other types of couplings beyond nonadiabatic ones. In the present study, the additional couplings were the spin-orbit couplings among the states $S_{0}$ to $S_{3}$ and $T_{1}$ to $T_{2}$. Within SHARC, these states are mixed to yield 'diagonal' states, on whose PESs the dynamics is carried out; this procedure includes calculating the gradients of the diagonal states from the spin-free gradients as well as propagating the electronic wavefunction using the spin-orbit Hamiltonian and the nonadiabatic couplings of the spin-free states. SHARC is available free of charge from http://sharc-md.org, and the development version from the authors upon request.

Initial conditions for the dynamics simulations were obtained by sampling from a quantum-harmonic Wigner distribution around the ground state minimum, based on a frequency calculation performed with the MRCIS method. 4,000 geometries were sampled from the distribution and for each geometry the vertical excitation energies and oscillator strengths were calculated, which were used to simulate an absorption spectrum (Supplementary Fig. 2) and to select the initial electronic state for the dynamics.

One hundred thirty seven trajectories were propagated for a total simulation time of up to $1 \mathrm{ps}$. The time step for the nuclear motion was $0.5 \mathrm{fs}$, while the electronic Schrödinger equation was integrated with a time step of $0.02 \mathrm{fs}$ using the local diabatization methodology ${ }^{63}$, which employs wavefunction overlaps ${ }^{64}$ to provide the information about the nonadiabatic couplings. An energy-based decoherence correction ${ }^{65}$ was applied to the electronic states in the diagonal basis. As stated above, the simulations were carried out on spin-adiabatic potentials, obtained by diagonalization of the spin-orbit Hamiltonian including states $S_{0}$ to $S_{3}$ and $T_{1}$ to $T_{2}$. However, for the analysis of the results the state populations were transformed back into the spin-free basis, since this allows for easier interpretation of the results. Note that the energies of $S_{4}, T_{3}$ and $T_{4}$ (but no gradients, spin-orbit couplings or wavefunction overlaps) were also calculated at each time step in order to scrutinize whether neglecting these states is justified. Furthermore, since the $S_{3}$ population decays within only 20 fs to the $S_{2}$ state, for all analysis and discussion purposes the $S_{3}$ population was merged into the $S_{2}$ population.

Wave function localization analysis. The calculations were performed using optimized geometries reported in the literature for cytosine ${ }^{48}$, uracil ${ }^{43,52}$, guanine $^{66}, 2$-thiouracil ${ }^{54}$ and 6 -thioguanine ${ }^{25,29}$, whereas for 2-thiocytosine the geometries reported here were used. For each molecule, the $n \pi^{*}$ and $\pi \pi^{*}$ excited-state minima and relevant CoIns were included. At each geometry, a CASSCF/ANO-L calculation was performed, averaging over 4 singlet states. For cytosine, uracil, 2-thiocytosine, and 2-thiouracil, a CAS $(14,10)$ was used, whereas for guanine and 6-thioguanine a $\operatorname{CAS}(18,13)$ was employed. These calculations were performed with MOLCAS ${ }^{60}$. The one-electron difference density matrices between the ground state and the first excited state were then analysed using the TheoDORE program ${ }^{42}$ in terms of the Mulliken detachment populations in order to arrive at the classification in Table 1. The Mulliken populations are given in Supplementary Table 5 .

Data availability. All data is available from the authors upon request.

\section{References}

1. Carell, T. et al. Structure and function of noncanonical nucleobases. Angew Chem. Int. Ed. Engl. 51, 7110-7131 (2012).

2. Carbon, J., David, H. \& Studier, M. H. Thiobases in Escherchia coli transfer RNA: 2-thiocytosine and 5-methylaminomethyl-2-thiouracil. Science 161, 1146-1147 (1968).

3. Zhang, S., Blain, J. C., Zielinska, D., Gryaznov, S. M. \& Szostak, J. W. Fast and accurate nonenzymatic copying of an RNA-like synthetic genetic polymer. Proc. Natl Acad. Sci. USA 110, 17732-17737 (2013).

4. Wei, F.-Y. et al. Deficit of tRNALys modification by cdkall causes the development of type 2 diabetes in mice. J. Clin. Invest. 121, 3598-3608 (2011).

5. Wojciechowski, F. \& Leumann, C. J. Alternative DNA base-pairs: from efforts to expand the genetic code to potential material applications. Chem. Soc. Rev. 40, 5669-5679 (2011).

6. Favre, A., Saintomé, C., Fourrey, J.-L., Clivio, P. \& Laugâa, P. Thionucleobases as intrinsic photoaffinity probes of nucleic acid structure and nucleic acid-protein interactions. J. Photochem. Photobiol. B 42, 109-124 (1998).

7. Wilhelmsson, L. M. Fluorescent nucleic acid base analogues. Q. Rev. Biophys. 43, 159-183 (2010).

8. Périgaud, C., Gosselin, G. \& Imbach, J. L. Nucleoside analogues as chemotherapeutic agents: a review. Nucleosides Nucleotides 11, 903-945 (1992).

9. Jordheim, L. P., Durantel, D., Zoulim, F. \& Dumontet, C. Advances in the development of nucleoside and nucleotide analogues for cancer and viral diseases. Nat. Rev. Drug Discov. 12, 447-464 (2013).

10. Attard, N. R. \& Karran, P. UVA photosensitization of thiopurines and skin cancer in organ transplant recipients. Photochem. Photobiol. Sci. 11, 62-68 (2012).

11. Pridgeon, S. W. et al. Thiothymidine combined with UVA as a potential novel therapy for bladder cancer. Br J. Cancer 104, 1869 (2011).

12. Pollum, M., Jockusch, S. \& Crespo-Hernández, C. E. Increase in the photoreactivity of uracil derivatives by doubling thionation. Phys. Chem. Chem. Phys. 17, 27851-27861 (2015).

13. Gemenetzidis, E., Shavorskaya, O., Xu, Y.-Z. \& Trigiante, G. Topical 4-thiothymidine is a viable photosensitiser for the photodynamic therapy of skin malignancies. J. Dermatol. Treat. 24, 209-214 (2013).

14. Trigiante, G. \& Xu, Y.-Z. In Hugo, A. G. (ed.) Photodynamic Therapy: Fundamentals, Applications and Health Outcomes 193-206 (Nova Science Publishers, 2015).

15. Pollum, M., Martínez-Fernández, L. \& Crespo-Hernández, C. E. In Barbatti, M., Borin, A. C. \& Ullrich, S. (eds) Photoinduced Phenomena in Nucleic Acids I, Vol 356 of Topics in Current Chemistry 245-327 (Springer Berlin Heidelberg, 2015).

16. Crespo-Hernández, C. E., Cohen, B., Hare, P. M. \& Kohler, B. Ultrafast excitedstate dynamics in nucleic acids. Chem. Rev. 104, 1977-2020 (2004).

17. Giussani, A., Segarra-Martí, J., Roca-Sanjuán, D. \& Merchán, M. Excitation of nucleobases from a computational perspective I: reaction paths. In Barbatti, M., Borin, A. C. \& Ullrich, S. (eds) Photoinduced Phenomena in Nucleic Acids I, Vol. 355 of Topics in Current Chemistry, 57-97 (Springer Berlin Heidelberg, 2015).

18. Reichardt, C. \& Crespo-Hernández, C. E. Ultrafast spin crossover in 4-thiothymidine in an ionic liquid. Chem. Commun. 46, 5963-5965 (2010).

19. Reichardt, C. \& Crespo-Hernández, C. E. Room-temperature phosphorescence of the DNA monomer analogue 4-thiothymidine in aqueous solutions after UVA excitation. J. Phys. Chem. Lett. 1, 2239-2243 (2010).

20. Reichardt, C., Guo, C. \& Crespo-Hernández, C. E. Excited-state dynamics in 6-thioguanosine from the femtosecond to microsecond time scale. J. Phys. Chem. B 115, 3263-3270 (2011).

21. Taras-Goślińska, K., Burdziński, G. \& Wenska, G. Relaxation of the $T_{1}$ excited state of 2-thiothymine, its riboside and deoxyriboside-enhanced nonradiative decay rate induced by sugar substituent. J. Photochem. Photobiol. A 275, 89-95 (2014).

22. Pollum, M. \& Crespo-Hernández, C. E. Communication: The dark singlet state as a doorway state in the ultrafast and efficient intersystem crossing dynamics in 2-thiothymine and 2-thiouracil. J. Chem. Phys. 140, 071101 (2014).

23. Pollum, M., Jockusch, S. \& Crespo-Hernández, C. E. 2,4-Dithiothymine as a potent UVA chemotherapeutic agent. J. Am. Chem. Soc. 136, 17930-17933 (2014).

24. Pollum, M., Ortiz-Rodríguez, L. A., Jockusch, S. \& Crespo-Hernández, C. E. The triplet state of 6-thio-2'-deoxyguanosine: Intrinsic properties and reactivity toward molecular oxygen. Photochem. Photobiol. 92, 286-292 (2016).

25. Martínez-Fernández, L., González, L. \& Corral, I. An ab initio mechanism for efficient population of triplet states in cytotoxic sulfur substituted DNA bases: the case of 6-thioguanine. Chem. Commun. 48, 2134-2136 (2012).

26. Gobbo, J. P. \& Borin, A. C. 2-thiouracil deactivation pathways and triplet states population. Comput. Theor. Chem. 1040-1041, 195-201 (2014).

27. Cui, G. \& Fang, W.-H. State-specific heavy-atom effect on intersystem crossing processes in 2-thiothymine: a potential photodynamic therapy photosensitizer. J. Chem. Phys. 138, 044315 (2013).

28. Cui, G. \& Thiel, W. Intersystem crossing enables 4-thiothymidine to act as a photosensitizer in photodynamic therapy: an ab initio QM/MM study. J. Phys Chem. Lett. 5, 2682-2687 (2014). 
29. Martínez-Fernández, L., Corral, I., Granucci, G. \& Persico, M. Competing ultrafast intersystem crossing and internal conversion: a time resolved picture for the deactivation of 6-thioguanine. Chem. Sci. 5, 1336-1347 (2014).

30. Pirillo, J., De Simone, B. C. \& Russo, N. Photophysical properties prediction of selenium- and tellurium-substituted thymidine as potential UVA chemotherapeutic agents. Theor. Chem. Acc. 135, 8 (2016).

31. Yu, H. et al. Internal conversion and intersystem crossing pathways in UV excited, isolated uracils and their implications in prebiotic chemistry. Phys. Chem. Chem. Phys. 18, 20168-20176 (2016).

32. Mai, S., Marquetand, P. \& González, L. Intersystem crossing pathways in the noncanonical nucleobase 2-thiouracil: a time-dependent picture. J. Phys. Chem. Lett. 7, 1978-1983 (2016).

33. Vetter, C., Wagner, C., Kaluerović, G. N., Paschke, R. \& Steinborn, D. Synthesis, characterization, and cytotoxicity of trimethylplatinum(IV) complexes with 2-thiocytosine and 1-methyl-2-thiocytosine ligands. Inorg. Chim. Acta 362, 189-195 (2009).

34. Shigeta, S. et al. Anti-herpesvirus activities and cytotoxicities of 2-thiopyrimidine nucleoside analogues in vitro. Antivir. Chem. Chemother. 10, 195-209 (1999).

35. Fillat, M. F. et al. Synthesis, structure and bactericide activity of (aminophosphane) gold(I) thiolate complexes. Eur. J. Inorg. Chem. 2011, 1487-1495 (2011).

36. Semenov, V. E. et al. Antimicrobial activity of pyrimidinophanes with thiocytosine and uracil moieties. Eur. J. Med. Chem. 46, 4715-4724 (2011).

37. Lozzio, C. B. \& Wigler, P. W. Cytotoxic effects of thiopyrimidines. J. Cell Physiol. 78, 25-31 (1971).

38. Mai, S., Marquetand, P. \& González, L. A general method to describe intersystem crossing dynamics in trajectory surface hopping. Int. J. Quantum Chem. 115, 1215-1231 (2015).

39. Crespo-Hernández, C. E. et al. Electronic and structural elements that regulate the excited-state dynamics in purine nucleobase derivatives. J. Am. Chem. Soc. 137, 4368-4381 (2015).

40. Maciejewski, A. \& Steer, R. P. The photophysics, physical photochemistry, and related spectroscopy of thiocarbonyls. Chem. Rev. 93, 67-98 (1993).

41. Gomzi, V. \& Herak, J. N. Ionization potentials of nucleobase analogs using partial third-order electron propagator method. J. Mol. Struct. 683, 155-157 (2004).

42. Plasser, F. TheoDORE: A package for theoretical density, orbital relaxation, and exciton analysis http://theodore-qc.sourceforge.net (2015).

43. Nakayama, A., Harabuchi, Y., Yamazaki, S. \& Taketsugu, T. Photophysics of cytosine tautomers: new insights into the nonradiative decay mechanisms from MS-CASPT2 potential energy calculations and excited-state molecular dynamics simulations. Phys. Chem. Chem. Phys. 15, 12322-12339 (2013).

44. Ismail, N., Blancafort, L., Olivucci, M., Kohler, B. \& Robb, M. A. Ultrafast decay of electronically excited singlet cytosine via a $\pi, \pi^{\star}$ to $n_{o}, \pi^{*}$ state switch. J. Am. Chem. Soc. 124, 6818-6819 (2002).

45. Merchán, M. \& Serrano-Andrés, L. Ultrafast internal conversion of excited cytosine via the lowest $\pi \pi^{*}$ electronic singlet state. J. Am. Chem. Soc. 125, 8108-8109 (2003)

46. Kistler, K. A. \& Matsika, S. Radiationless decay mechanism of cytosine: an $a b$ initio study with comparisons to the fluorescent analogue 5-methyl-2pyrimidinone. J. Phys. Chem. A 111, 2650-2661 (2007).

47. Hudock, H. R. \& Martínez, T. J. Excited-state dynamics of cytosine reveal multiple intrinsic subpicosecond pathways. ChemPhysChem. 9, 2486-2490 (2008).

48. Barbatti, M., Aquino, A. J. A., Szymczak, J. J., Nachtigallová, D. \& Lischka, H. Photodynamical simulations of cytosine: characterization of the ultrafast bi-exponential UV deactivation. Phys. Chem. Chem. Phys. 13, 6145-6155 (2011).

49. Mai, S., Marquetand, P., Richter, M., González-Vázquez, J. \& González, L. Singlet and triplet excited-state dynamics study of the keto and enol tautomers of cytosine. ChemPhysChem. 14, 2920-2931 (2013).

50. Yamazaki, S. \& Taketsugu, T. Nonradiative deactivation mechanisms of uracil, thymine, and 5-fluorouracil: A comparative ab initio study. J. Phys. Chem. A 116, 491-503 (2012).

51. Merchán, M. et al. Unified model for the ultrafast decay of pyrimidine nucleobases. J. Phys. Chem. B 110, 26471-26476 (2006).

52. Nachtigallová, D. et al. Nonadiabatic dynamics of uracil: population split among different decay mechanisms. J. Phys. Chem. A 115, 5247-5255 (2011).

53. Richter, M., Mai, S., Marquetand, P. \& González, L. Ultrafast intersystem crossing dynamics in uracil unravelled by ab initio molecular dynamics. Phys. Chem. Chem. Phys. 16, 24423-24436 (2014).

54. Mai, S., Marquetand, P. \& González, L. A static picture of the relaxation and intersystem crossing mechanisms of photoexcited 2-thiouracil. J. Phys. Chem. A 119, 9524-9533 (2015).

55. Heß, B. A., Marian, C. M., Wahlgren, U. \& Gropen, O. A mean-field spin-orbit method applicable to correlated wavefunctions. Chem. Phys. Lett. 251, 365-371 (1996).

56. Reichardt, C., Vogt, R. A. \& Crespo-Hernández, C. E. On the origin of ultrafast nonradiative transitions in the nitro-polycyclic aromatic hydrocarbons: excited-state dynamics in 1-nitronaphthalene. J. Chem. Phys. 131, 224518 (2009).
57. Levine, B. G., Coe, J. D. \& Martínez, T. J. Optimizing conical intersections without derivative coupling vectors: Application to multistate multireference Second-Order perturbation theory (MS-CASPT2). J. Phys. Chem. B 112, 405-413 (2008).

58. Bearpark, M. J., Robb, M. A. \& Schlegel, H. B. A direct method for the location of the lowest energy point on a potential surface crossing. Chem. Phys. Lett. 223, 269 (1994).

59. Neese, F. The ORCA program system. WIREs Comput. Mol. Sci. 2, 73-78 (2012)

60. Aquilante, F. et al. Molcas 7: The next generation. J. Comput. Chem. 31, 224-247 (2010).

61. Werner, H., Knowles, P. J., Knizia, G., Manby, F. R. \& Schütz, M. Molpro: A general-purpose quantum chemistry program package. WIREs Comput. Mol. Sci. 2, 242-253 (2012).

62. Lischka, H. et al. Columbus-a program system for advanced multireference theory calculations. WIREs Comput. Mol. Sci. 1, 191-199 (2011).

63. Granucci, G., Persico, M. \& Toniolo, A. Direct semiclassical simulation of photochemical processes with semiempirical wave functions. J. Chem. Phys. 114, 10608-10615 (2001).

64. Pittner, J., Lischka, H. \& Barbatti, M. Optimization of mixed quantum-classical dynamics: time-derivative coupling terms and selected couplings. Chem. Phys. 356, 147-152 (2009).

65. Granucci, G. \& Persico, M. Critical appraisal of the fewest switches algorithm for surface hopping. J. Chem. Phys. 126, 134114 (2007).

66. Serrano-Andrés, L., Merchán, M. \& Borin, A. C. A three-state model for the photophysics of guanine. J. Am. Chem. Soc. 130, 2473-2484 (2008).

67. Kotur, M., Weinacht, T., Zhou, C. \& Matsika, S. Following ultrafast radiationless relaxation dynamics with strong field dissociative ionization: a comparison between adenine, uracil, and cytosine. IEEE J. Sel. Top. Quant. Electron. 18, 187-194 (2012).

68. Ullrich, S., Schultz, T., Zgierski, M. Z. \& Stolow, A. Electronic relaxation dynamics in DNA and RNA bases studied by time-resolved photoelectron spectroscopy. Phys. Chem. Chem. Phys. 6, 2796-2801 (2004).

69. Matsika, S., Spanner, M., Kotur, M. \& Weinacht, T. C. Ultrafast relaxation dynamics of uracil probed via strong field dissociative ionization. J. Phys. Chem. A 117, 12796-12801 (2013).

70. Canuel, C. et al. Excited states dynamics of DNA and RNA bases: characterization of a stepwise deactivation pathway in the gas phase. J. Chem. Phys. 122, 074316 (2005)

\section{Acknowledgements}

S.M., P.M. and L.G. thank the Austrian Science Fund (FWF) through project P25827, the COST action CM1204 (XLIC) and the Vienna Scientific Cluster (VSC) for the allocation of computational time. We also thank F. Plasser for assistance with the TheoDORE program. I.C. and L.M.-F. thank the Comunidad Autónoma de Madrid, the Ministerio de Economía y Competitividad (Spain) for an FPU (L.M.-F.) grant, the Projects FOTOCARBON-CM S2013/MIT-2841 and No. CTQ2015-63997-C2, and the ERA-Chemistry Project PIM2010EEC-00751 for financial support, as well as the Centro de Computación Científca UAM for generous allocation of computational time. M.P., N.D. and C.E.C.-H. acknowledge the CAREER program of the National Science Foundation (Grant No. CHE-1255084) for financial support.

\section{Author contributions}

C.E.C.-H., I.C. and L.G. conceived the research. M.P., N.D. and C.E.C.-H. performed al spectroscopic experiments. L.M.-F. and I.C. performed the static quantum chemistry calculations. S.M., P.M., and L.G. performed the dynamics simulations and the calculations related to Table 1 . All authors discussed and contributed to the manuscript.

\section{Additional information}

Supplementary Information accompanies this paper at http://www.nature.com/ naturecommunications

Competing financial interests: The authors declare no competing financial interests.

Reprints and permission information is available online at http://npg.nature.com/ reprintsandpermissions/

How to cite this article: Mai, S. et al. The origin of efficient triplet state population in sulfur-substituted nucleobases. Nat. Commun. 7, 13077 doi: 10.1038/ncomms13077 (2016)

This work is licensed under a Creative Commons Attribution 4.0 International License. The images or other third party material in this
article are included in the article's Creative Commons license, unless indicated otherwise in the credit line; if the material is not included under the Creative Commons license, users will need to obtain permission from the license holder to reproduce the material. To view a copy of this license, visit http://creativecommons.org/licenses/by/4.0/

(C) The Author(s) 2016 